\title{
Design of Speed Optimized Analog to Digital Converter using VHDL
}

\author{
Kondamudi Kishore Babu ${ }^{1}$, B.Raghavaiah ${ }^{2}$ \\ ${ }^{I}$ PG Student (M. Tech), Dept. of ECE, Chirala Engineering College, Chirala., A.P, India. \\ ${ }^{2}$ Associate Professor, Dept. of ECE, Chirala Engineering College, Chirala., A.P, India.
}

\begin{abstract}
An analog-to-digital converter (abbreviated $A D C, A / D$ or $A$ to $D$ ) is a device that converts a continuous quantity to a discrete time digital representation. An ADC may also provide an isolated measurement. The reverse operation is performed by a digital-to-analog converter (DAC). Typically, an ADC is an electronic device that converts an input analog voltage or current to a digital number proportional to the magnitude of the voltage or current. However, some non-electronic or only partially electronic devices, such as rotary encoders, can also be considered ADCs.

In order to increase the flexibility of control for ADC, a new control method for ADC based on FPGA is proposed in this paper. A state transition diagram can be drawn according to the timing diagram of ADC, the design is implemented using Very High-speed Integrated Circuit Hardware Description Language (VHDL). The operations of ADC are simulated using the modelsim tool and ADC design is synthesized using Xilinx tool.
\end{abstract}

\section{Introduction}

In general converter especially telecommunication converter is used for converting a signal from one frequency to another or it is a device for changing one substance or form or state into another or telecommunications systems used in transmitting messages over a distance electronically.

Here we have many converters mainly we see only

- Analog to Digital Converter

- Digital to Analog Converter

- Successive Analog to Digital Converter

A signal as referred to in communication systems, signal processing, and electrical engineering "is a function that conveys information about the behavior or attributes of some phenomenon". In the physical world, any quantity exhibiting variation in time or variation in space (such as an image) is potentially a signal that might provide information on the status of a physical system, or convey a message between observers, among other possibilities. The IEEE Transactions on Signal Processing elaborates upon the term "signal" as follows The term "signal" includes, among others, audio, video, speech, image, communication, geophysical, sonar, radar, medical and musical signals. Other examples of signals are the output of a thermocouple, which conveys temperature information, and the output of a $\mathrm{pH}$ meter which conveys acidity information. Typically, signals are often provided by asensor, and often the original form of a signal is converted to another form of energy using a transducer. For example, a microphone converts an acoustic signal to a voltage waveform, and a speaker does the reverse.

The formal study of the information content of signals is the field of information theory. The information in a signal is usually accompanied by noise. The term noise usually means an undesirable random disturbance, but is often extended to include unwanted signals conflicting with the desired signal (such as crosstalk). The prevention of noise is covered in part under the heading of signal integrity. The separation of desired signals from a background is the field of signal recovery, one branch of which is estimation theory, a probabilistic approach to suppressing random disturbances.

Engineering disciplines such as electrical engineering have led the way in the design, study, and implementation of systems involving transmission, storage, and manipulation of information. In the latter half of the 20th century, electrical engineering itself separated into several disciplines, specializing in the design and analysis of systems that manipulate physical signals; electronic engineering and computer engineering as examples; while design engineering developed to deal with functional design of man-machine interfaces.

An analog or analogue signal is any continuous signal for which the time varying feature (variable) of the signal is a representation of some other time varying quantity, i.e., analogous to another time varying signal. 


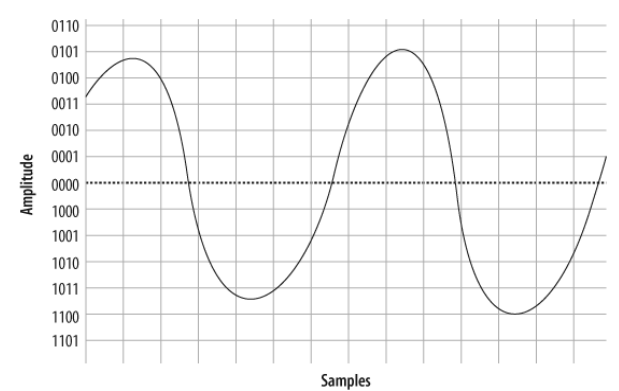

Figure 1 Example of an Analog Signal

For example, in an analog audio signal, the instantaneous voltage of the signal varies continuously with the pressure of the sound waves. It differs from a digital signal, in which a continuous quantity is represented by a discrete function which can only take on one of a finite number of values. The term analog signal usually refers to electrical signals; however, mechanical, pneumatic, hydraulic, and other systems may also convey analog signals.

Any information may be conveyed by an analog signal; often such a signal is a measured response to changes in physical phenomena, such as sound, light, temperature, position, or pressure. The physical variable is converted to an analog signal by a transducer. For example, in sound recording, fluctuations in air pressure (that is to say, sound) strike the diaphragm of a microphone which induces corresponding fluctuations in the current produced by a coil in an electromagnetic microphone, or the voltage produced by a condenser microphone. The voltage or the current is said to be an "analog" of the sound.

A digital signal is a physical signal that is a representation of a sequence of discrete values (a quantified discrete-time signal), for example of an arbitrary bit stream, or of a digitized (sampled and analog-to-digital converted) analog signal. The term digital signal can refer to either of the following:

- any continuous-time waveform signal used in digital communication, representing a bit stream or other sequence of discrete values

- a pulse train signal that switches between a discrete number of voltage levels or levels of light intensity, also known as a line coded signal or baseband transmission, for example a signal found in digital electronics or in serial communications, or a pulse code modulation (PCM) representation of a digitized analog signal. Digital signal

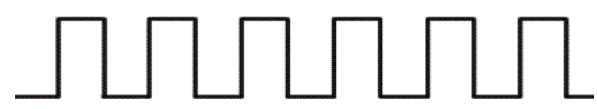

Figure 2 An example of a Digital Signal

A signal that is generated by means of a digital modulation method (digital passband transmission), to be transferred between modems, is in the first case considered as a digital signal, and in the second case as converted to an analog signal.

\section{Analog to Digital Converter}

An ADC is defined by its bandwidth (the range of frequencies it can measure) and its signal to noise ratio (how accurately it can measure a signal relative to the noise it introduces). The actual bandwidth of an ADC is characterized primarily by its sampling rate, and to a lesser extent by how it handles errors such as aliasing. The dynamic range of an ADC is influenced by many factors, including the resolution (the number of output levels it can quantize a signal to), linearity and accuracy (how well the quantization levels match the true analog signal) and jitter (small timing errors that introduce additional noise). The dynamic range of an ADC is often summarized in terms of its effective number of bits (ENOB), the number of bits of each measure it returns that are on average not noise.

An ideal ADC has an ENOB equal to its resolution. ADCs are chosen to match the bandwidth and required signal to noise ratio of the signal to be quantized. If an $\mathrm{ADC}$ operates at a sampling rate greater than twice the bandwidth of the signal, then perfect reconstruction is possible given an ideal ADC and neglecting quantization error. The presence of quantization error limits the dynamic range of even an ideal ADC, however, if the dynamic range of the ADC exceeds that of the input signal, its effects may be neglected resulting in an essentially perfect digital representation of the input signal. 


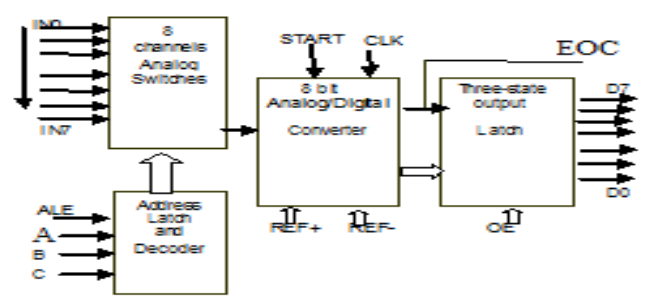

Figure 3 Block diagram of ADC

\section{Analog Switch:}

Analog Switches TI's analog switches are designed to pass (or isolate) analog signals (both voltage and current) and support analog applications such as audio and video data transmission. TI analog switches are available in a wide range of voltages (from 0.8 to $12 \mathrm{~V}$ ), support fast data throughput (up to 2-GHz bandwidth) and offer low on-resistance and input capacitance for Decreased signal distortion and insertion loss. TI analog switches are available in the TI Switch (TS) technology family. The TS product family encompasses a variety of analog switches with different $\mathrm{ON}$ resistances, bandwidth, charge injection, and total harmonic distortion to target any application.

\section{SAR Architecture}

\section{Application of an ADC}

Successive approximation employs a binary search algorithm in a feedback loop including a 1 bit A/D converter. The Fig. 3 illustrates this architecture which consists of a front end track \& hold circuit, comparator, DAC and SAR logic. SAR logic is basically a shift register combined with decision logic and decision register. The pointer points to the last bit changed in the decision register and the data stored in this register is the result of all comparisons performed during conversion period. During binary search, the circuit halves the difference between the sampled signal ( VIN ) and DAC output (VDAC). The conversion first sets MSB as 1 so that the DAC produces midscale at analog output.

The comparator is then strobed to determine the polarity of $V_{I N} \square V_{D A C}$. The pointer and the decision logic direct to logical output of the comparator to the MSB. If $V_{I N}>V_{D A C}$, the MSB of the register is maintained at 1 or else set to 0 . Subsequently the pointer is set to choose the bit penultimate to MSB as 1 . After the DAC output has settled to its new value, the comparator is strobed once again and the above sequence is repeated.

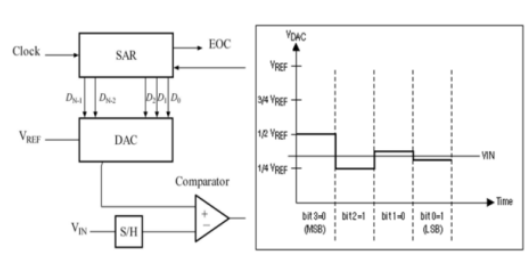

Figure 4 Block diagram of SAR ADC \& DAC Output Waveform

For a resolution of $\mathrm{M}$ bits, the successive approximation architecture is at least Mtimes slower than the full-flash configurations, but it offers several advantages.

- The comparator offset voltage does not affect the overall linearity of the converter because it can be represented as a voltage source in series with Sample \& hold output, indicating that offset voltage simply adds to analog input and hence appears as an offset in the overall characteristics. Consequently the comparator can be designed for high speed operation in high resolution systems.

- This architecture does not require an explicit subtractor which is an important advantage for high resolution applications

- The circuit complexity and power dissipation are in general less than that of the other architectures.

If the Sample \& hold circuit provides the required linearity, speed and comparator input referred noise is small enough, and then the converteres performance depends primarily on the DAC. In particular differential and integral non linearities of the converter are given by those of the DAC, and the maximum conversion rate is limited by its output settling time. In the first conversion cycle, the DAC output must settle to maximum resolution of the system so that the comparator determines the MSB correctly.

If the clock period is constant, the following conversion cycles will be as long as the first one, implying that the conversion rate is constrained by the speed of the DAC. 


\section{Track \& Hold}

A sample-and-hold $(\mathrm{S} / \mathrm{H})$ or track-and-hold $(\mathrm{T} / \mathrm{H})$ circuit is frequently required to capture rapidly varying signals for subsequent processing by slower circuitry. The function of the $\mathrm{S} / \mathrm{H}$ circuit is to track/sample the analog input signal and to hold that value while subsequent circuitry digitizes it. Although an $\mathrm{S} / \mathrm{H}$ refers to a device which spends an infinitesimal time acquiring signals and a $\mathrm{T} / \mathrm{H}$ refers to a device which spends a finite time in this mode, common practice will be followed and the two terms will be used interchangeably throughout this discussion as will the terms sample and track.

The function of a track-and-hold circuit is to buffer its input signal accurately during track mode providing at its output a signal which is linearly proportional to the input, and to maintain a constant output level during hold mode equal to the $\mathrm{T} / \mathrm{H}$ output value at the instant it was strobed from track to hold by an external clock signal. Fig. 4 shows the waveforms of a practical sample-and-hold circuit.

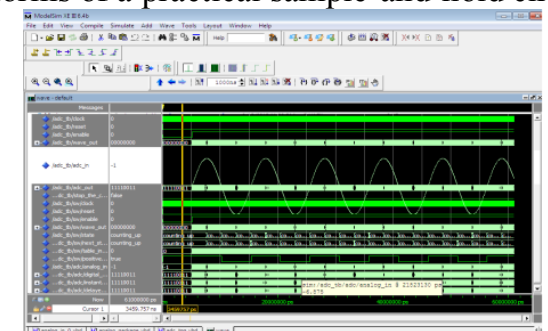

\section{Figure 5 ADC Simulation Waveform}

\section{Conclusions}

Xilinx and Modelsim softwares includes a simulator which can be used to simulate the behavior and performance of circuits designed for implementation in programmable logic devices. The simulator allows the user to apply test vectors as inputs to the designed circuit and to observe the outputs generated in response. In addition to being able to observe the simulated values on the $\mathrm{I} / \mathrm{O}$ pins of the circuit, it is also possible to probe the internal nodes in the circuit. The simulator makes use of the Waveform Editor, which makes it easy to represent the desired signals as waveforms. According to the results of simulation, a new control method for $\mathrm{ADC}$ is achieved. Advantages of this new method are simple programming method for high Clock frequency.

\section{Acknowledgements}

The authors would like to thank the anonymous reviewers for their comments which were very helpful in improving the quality and presentation of this paper.

\section{References:}

[1] Petouris M (Petouris, M.), Kalantzopoulos A (Kalantzopoulos, A.),Zigouris E (Zigouris, E.), “An FPGA-based Digital Camera System Controlled from an LCD Touch Panel" INTERNATIONAL SYMPOSIUM ON SIGNALS, CIRCUITS AND SYSTEMS, pp. 241- 244, 2009.

[2] El-Medany, W.M. ; Hussain, M.R, “A FPGA-based advanced real traffic light controller system design” 007 4th IEEE Workshop on Intelligent Data Acquisition and Advanced Computing Systems: Technology and Applications, IDAACS, pp. 100-105, 2007.

[3] Guo, Jianmin, Zhang, Ke, Kong, Ming, Li, Wenhong, "Novel ADC architecture for digital voltage regulator module controllers," Pan Tao Ti Hsueh Pao/Chinese Journal of Semiconductors, v 27, n 12, p 2112-2117,December 2006

[4] Hussein, Aziza I. ,Gruenbacher, Don M.; Ibrahim, Noureddin M, "Design and verification techniques used in a graduate level VHDLcourse," Proceedings - Frontiers in Education Conference, v 2, p 13a4-28 - 13a4-31, 1999.

\section{Authors Profile:}

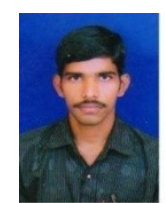

Kondamudi Kishore Babu is Pursuing his M. Tech from Chirala Engineering College, Chirala in the department of Electronics \& Communications Engineering (ECE) with specialization in VLSI \& Embedded systems.

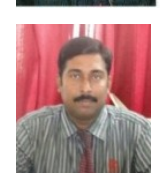

B.RAGHAVAIAH is working as an Associate Professor in the department of Electronics \& Communication Engineering in Chirala Engineering College, Chirala. He has completed masters from JNTUK. He has over 8 years of teaching experience. 